\title{
FAST ALGORITHM FOR PERIODIC DENSITY FITTING FOR BLOCH WAVES
}

\author{
JIANFENG LU AND LEXING YING
}

\begin{abstract}
We propose an efficient algorithm for density fitting of Bloch waves for Hamiltonian operators with periodic potential. The algorithm is based on column selection and random Fourier projection of the orbital functions. The computational cost of the algorithm scales as $\mathscr{O}\left(N_{\text {grid }} N^{2}+\right.$ $N_{\text {grid }} N K \log (N K)$ ), where $N_{\text {grid }}$ is number of spatial grid points, $K$ is the number of sampling $k$ points in first Brillouin zone, and $N$ is the number of bands under consideration. We validate the algorithm by numerical examples in both two and three dimensions.
\end{abstract}

\section{INTRODUCTION}

Consider a Hamiltonian operator with periodic potential

$$
H=-\frac{1}{2} \Delta+V
$$

For simplicity of notation, we assume without loss of generality that $V$ is periodic with respect to $\mathbb{Z}^{d}$, where $d$ is the spatial dimension: i.e., $V\left(x+e_{i}\right)=V(x)$ for $\left\{e_{i}, i=1, \ldots, d\right\}$ the set of standard Cartesian basis vectors of $\mathbb{R}^{d}$. From the standard Bloch-Floquet theory (see e.g., [13]), the spectrum of the operator $H$ is given by the Bloch eigenvalue problem on the unit cell $\Gamma=[0,1)^{d}$ :

$$
\begin{aligned}
& \left(-\frac{1}{2} \Delta+V\right) \psi_{n, k}=E_{n, k} \psi_{n, k}, \quad \text { on } \Gamma ; \\
& e^{-i(k \cdot x)} \psi_{n, k}(x) \quad \text { is periodic on } \Gamma .
\end{aligned}
$$

Here $k \in \Gamma^{*}=[-\pi, \pi)^{d}$ is known as the crystal momentum, where $\Gamma^{*}$ is called the first Brillouin zone (FBZ), which is the unit cell of the reciprocal lattice. The eigenfunction $\psi_{n, k}$ for $n \in \mathbb{N}$ and $k \in \Gamma^{*}$ are called Bloch waves. We extend them from $\Gamma$ to the whole $\mathbb{R}^{3}$ using the Bloch-periodicity (2b). It would be convenient to introduce the periodic part of the Bloch function, denoted as

$$
u_{n, k}(x)=e^{-i(k \cdot x)} \psi_{n, k}(x), \quad x \in \mathbb{R}^{d} .
$$

By definition, $u_{n, k}$ is periodic with respect to $\mathbb{Z}^{d}$.

In this work, we are interested in the pair density $\bar{u}_{n, k}(x) u_{m, l}(x)$ for $n, m \in \mathbb{N}, k, l \in \Gamma^{*}$, and $x \in \Gamma$. In particular, we aim at an approximation of the pair density of the type

$$
\bar{u}_{n, k}(x) u_{m, l}(x) \approx \sum_{\mu} \bar{C}_{n, k}^{\mu} C_{m, l}^{\mu} P_{\mu}(x),
$$

Date: November 7, 2018.

The work of J.L. is supported in part by the Alfred P. Sloan Foundation and the National Science Foundation under grant DMS-1312659 and DMS-1454939. The work of L.Y. is partially supported by the National Science Foundation under grant DMS-1521830 and the U.S. Department of Energy's Advanced Scientific Computing Research program under grant DE-FC02-13ER26134/DE-SC0009409. We would like to thank helpful discussions with Volker Blum and Xinguo Ren. 
where $\left\{P_{\mu}\right\}$ is a set of auxiliary basis functions we use to expand the pair density and $C_{n, k}^{\mu}$ gives the expansion coefficients. In terms of the Bloch waves, the approximation is then

$$
\bar{\psi}_{n, k}(x) \psi_{m, l}(x) \approx \sum_{\mu} \bar{C}_{n, k}^{\mu} C_{m, l}^{\mu} P_{\mu}(x) e^{i(l-k) \cdot x} .
$$

The approximation of pair density in the form of

$$
\bar{u}_{n, k}(x) u_{m, l}(x) \approx \sum_{\mu} T_{n k, m l}^{\mu} P_{\mu}(x)
$$

is known as periodic density fitting or density fitting for crystals in the literature [6 6 | 10 . Note that the approximation (4) is a special form, in which the expansion coefficient $T_{n k, m l}^{\mu}=\bar{C}_{n, k}^{\mu} C_{m, l}^{\mu}$ has a separable structure. The periodic density fitting is a generalization of the conventional density fitting for molecules (see e.g., 10 14 $15,18-20 \mid$ ), which has wide applications in electronic structure calculations. In density fitting, the pair density for a set of orbital functions $\left\{\psi_{n}\right\}$ (without the $k$-dependence) is approximated as

$$
\bar{\psi}_{n}(x) \psi_{m}(x) \approx \sum_{\mu} T_{n m}^{\mu} P_{\mu}(x) .
$$

Here with slight abuse of notation, we use again $T$ to denote the expansion coefficients.

Conventionally in density fitting, a set of auxiliary functions $P_{\mu}(x)$ are chosen, for example as Gaussian functions or atom centered orbital functions, the expansion coefficients $T$ are obtained using least square fitting with respect to a suitable metric $\left(L^{2}\right.$ or Coulomb metric, see Section 3 below). The computational cost to obtain these coefficients scales as $\mathscr{O}\left(N^{4}\right)$, where $N$ is the total number of orbital functions. Moreover, the conventional procedure does not yield the separable structure, which is useful to reduce the computational cost for electronic structure calculations, see e.g., \begin{tabular}{l|l|l|l|l|l|l|l|l|l|l|l|l}
3 & 4 & 11 & 12 & 16 & 17 &
\end{tabular}.

In our previous work 9 , we developed a $\mathscr{O}\left(N_{\text {grid }} N^{2} \log N\right)$ scaling algorithm for density fitting without the $k$ point dependence, where $N_{\text {grid }}$ is number of spatial grids and $N$ is the number of orbital functions. The novelty is to recast the density fitting problem as a column selection procedure: Instead of fixing the auxiliary basis functions and compute the expansion coefficients, we look for a collection of grid points $\left\{x_{\mu}\right\}$ that well represent the pair densities, such that the auxiliary basis functions follow as a least square fitting.

$$
\bar{\psi}_{m}(x) \psi_{n}(x) \approx \sum_{\mu} \bar{\psi}_{m}\left(x_{\mu}\right) \psi_{n}\left(x_{\mu}\right) P_{\mu}(x) .
$$

This work extends our approach to periodic density fitting of Bloch waves. In order to further reduce the computational cost, we use a more efficient scheme for random projection based on the tensor product structure of the pair density $\rho$. Our resulting density fitting scheme scales as $\mathscr{O}\left(N_{\text {grid }} N^{2}+N_{\text {grid }} N K \log (N K)\right)$, where $N_{\text {grid }}$ is number of spatial grid points, $K$ is the number of $k$-points in first Brillouin zone, and $N$ is the number of bands under consideration. Recall that in the periodic case, the total number of Bloch waves is given by $N K$. Note that if we take $K=1$ so that we go back to the situation considered in [9], our current algorithm costs as $\mathscr{O}\left(N_{\text {grid }} N^{2}\right)$ and is hence more efficient than our previous algorithm. Moreover, the computational cost scales 
almost linearly with respect to the increase of $K$, with a "quadratic" coefficient $\mathscr{O}\left(N_{\text {grid }} N\right)$; in fact, in practice, the actual running time is dominated by the $\mathscr{O}\left(N_{\text {grid }} N^{2}\right)$ term, independent of $K$.

\section{Algorithms}

Let us consider a set of given orbitals $u_{n, k}(x)$ for $n=1, \ldots, N, k \in \mathscr{K}$ a discretization of the first Brillouin zone with $|\mathscr{K}|=K$, and $x \in \mathscr{X}$ a grid in the unit cell with $|\mathscr{X}|=N_{\text {grid. }}$. Similar to our previous work [9], the basic idea is to select a suitable subset of grid points to represent the pair densities $\rho_{n k m l}(x)=\bar{u}_{n, k}(x) u_{m, l}(x)$.

$$
\rho_{n k m l}(x) \approx \sum_{\mu} \rho_{n k m l}\left(x_{\mu}\right) P_{\mu}(x)=\sum_{\mu} \bar{u}_{n, k}\left(x_{\mu}\right) u_{m, l}\left(x_{\mu}\right) P_{\mu}(x) .
$$

After the grid points $\left\{x_{\mu}\right\}$ are determined, the auxiliary basis functions $P_{\mu}$ follow from a least square fitting.

Note that if we view $\rho$ as a matrix of dimension $N^{2} K^{2} \times N_{\text {grid }}$ with $(n k m l)$ being the row index and $x$ being the column index, the choice of $x_{\mu}$ amounts to select a sub-collection of the columns to represent all the matrix columns. One immediate approach for the column selection is to use a pivoted QR algorithm [2] on $\rho$. For completeness, we recall the column selection algorithm based on pivoted QR algorithm here.

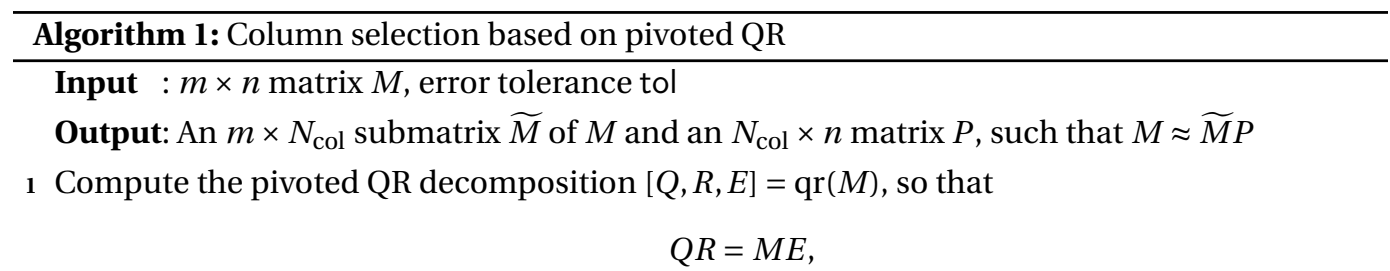

where $E$ is an $n \times n$ permutation matrix, $Q$ is an $m \times m$ unitary matrix and $R$ is an $m \times n$ upper triangular matrix with diagonal entries in decreasing order;

2 Set $N_{\text {col }}$ such that

$$
R_{N_{\mathrm{col}}, N_{\mathrm{col}}} \geq \mathrm{tol} \cdot R_{1,1}>R_{N_{\mathrm{col}}+1, N_{\mathrm{col}}+1}
$$

3 Set $\widetilde{M}=(M E)_{: 1: 1: N_{\text {col }}}$, the first $N_{\text {col }}$ columns of $M E$, where Matlab notations are used for submatrices. Note that $M E$ amounts to a permutation of the columns of $M$.

4 Compute $P=R_{1: N_{\mathrm{col}}, 1: N_{\mathrm{col}}}^{-1} R_{1: N_{\mathrm{col}}:} E^{-1}$.

The computational cost of directly applying Algorithm 1 on the matrix $\rho$ is however prohibitively expensive, since the computational complexity of the QR step scales as $\mathscr{O}\left(N_{\text {grid }} N^{4} K^{4}\right)$ (recall that the matrix $\rho$ has dimension $N^{2} K^{2} \times N_{\text {grid }}$ ).

In order to reduce the complexity, it is thus crucial to apply a random projection on the matrix $\rho$, in the spirit of the pioneering works 7 [2] 21 . The idea is to find an alternative representation of the column space of $\rho$ by "compressing" the size of the matrix for the pivoted QR algorithm. In our previous work [9], we apply a random Fourier projection on $\rho$, which amounts to taking a sample of random linear combinations of rows of the $\rho$ matrix:

$$
\varrho_{\xi}(x)=\sum_{\gamma} e^{-i 2 \pi \gamma \xi\left(N^{2} K^{2}\right)^{-1}} \eta_{\gamma} \rho_{\gamma}(x)
$$


for random samples of $\xi \in\left\{1, \ldots, N^{2} K^{2}\right\}$ of total number proportional to $N$ and $\eta_{\gamma}$ being random unit complex number for each $\gamma$. Here, we have used $\gamma \in\left\{1, \ldots, N^{2} K^{2}\right\}$ to denote the row index $(\mathrm{nkml})$ for simplicity of notations. Fixing $\xi, \varrho_{\xi}$ is a random linear combination of the rows $\rho_{\gamma}$. Thus, important columns of $\rho$ remains important columns of $\varrho$ with high probability.

The total size of the sample $\xi$ to guarantee successful identification of important columns is $\mathscr{O}(N)$ and is much smaller than the original number of rows of $\rho$, and hence the cost of the pivoted QR algorithm is much reduced. The computational cost is dominated by using FFT to compute [10, and is hence $\mathscr{O}\left(N_{\text {grid }}(N K)^{2} \log (N K)\right)$.

While the efficiency is much improved by using the algorithm in [9], it is still expensive in the current context due to the large number of rows of the matrix $\rho$. Our key observation to further reduce the computational cost is that $\rho$ given by $\rho_{n k m l}(x)=\bar{u}_{n, k}(x) u_{m, l}(x)$ has the tensor product structure, in the sense that the row index $(\mathrm{nkml})$ is naturally separated into two groups $(n k)$ and $(\mathrm{ml})$. We may explore this structure by taking the random Fourier projection on $u$ instead, which has a much smaller dimension than $\rho$, and hence makes the row projection more efficient. More concretely, we consider instead a projection of the type (cf. [10)

$$
M_{i j}(x)=\sum_{\alpha} e^{i 2 \pi \alpha \xi_{i}(N K)^{-1}} \bar{\eta}_{\alpha} \bar{u}_{\alpha}(x) \sum_{\beta} e^{-i 2 \pi \beta \xi_{j}(N K)^{-1}} \eta_{\beta} u_{\beta}(x)
$$

for $\left\{\xi_{i}\right\} \subset\{1, \ldots, N K\}$ and $\alpha, \beta \in\{1, \ldots, N K\}$ being the row index of $u$, viewed as a $(N K) \times N_{\text {grid }}$ matrix. Note that we can rewrite (11) as

$$
M_{i j}(x)=\sum_{\alpha, \beta} e^{i 2 \pi\left(\alpha \xi_{i}-\beta \xi_{j}\right)(N K)^{-1}} \bar{\eta}_{\alpha} \eta_{\beta} \bar{u}_{\alpha}(x) u_{\beta}(x)=\sum_{\alpha, \beta} e^{i 2 \pi\left(\alpha \xi_{i}-\beta \xi_{j}\right)(N K)^{-1}} \bar{\eta}_{\alpha} \eta_{\beta} \rho_{\alpha \beta}(x) .
$$

and hence this still corresponds to a random linear combination of the rows of $\rho$, and hence could be still used to select important columns of $\rho$ to represent the column space. The details of the algorithm are described in Algorithm2

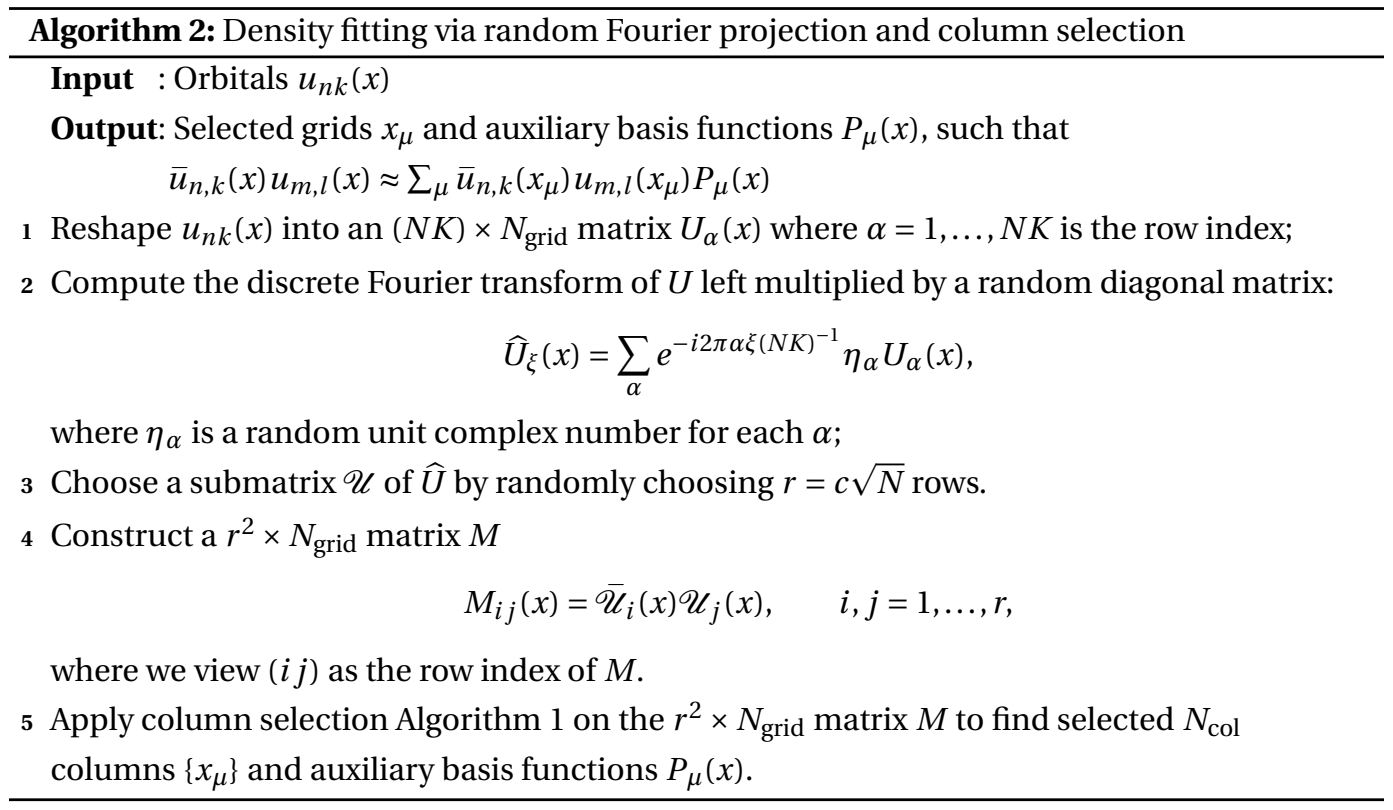


The computationally costly steps in Algorithm 2 are:

(1) Step 2 requires FFT of vectors of length $(N K)$ for $N_{\text {grid }}$ times, and hence has computational cost $\mathscr{O}\left(N_{\text {grid }} N K \log (N K)\right)$;

(2) Step 4 scales as $\mathscr{O}\left(r^{2} N_{\text {grid }}\right)=\mathscr{O}\left(N N_{\text {grid }}\right)$ by the choice of $r=c \sqrt{N}$;

(3) Step 5 requires pivoted QR algorithm applied on a $N \times N_{\text {grid }}$ matrix, and hence has cost $\mathscr{O}\left(N^{2} N_{\text {grid }}\right)$, which dominates the other steps in Algorithm 1

Therefore, the total computational cost of the algorithm scales as $\mathscr{O}\left(N_{\text {grid }} N^{2}+N_{\text {grid }} N K \log (N K)\right)$. In practice, the prefactor of the FFT is much smaller than the one of the pivoted QR factorization. As a result, the $\mathscr{O}\left(N_{\text {grid }} N^{2}\right)$ part dominates the actual running time.

\section{NUMERICAL EXAMPLES}

In this section, we consider a few model examples to demonstrate the effectiveness of the algorithms proposed above. In order to measure the error of the density fitting, two metrics can be used. The error in $L^{2}$ metric measures

$$
\left\|\rho_{n k m l}-\widetilde{\rho}_{n k m l}\right\|_{2},
$$

for $\widetilde{\rho}_{n k m l}=\sum_{\mu} \bar{C}_{n k}^{\mu} C_{m l}^{\mu} P_{\mu}$, while the error in Coulomb metric is given by

$$
\left\|\rho_{n k m l}-\widetilde{\rho}_{n k m l}\right\|_{C}=\left(\iint_{\Gamma \times \Gamma}\left(\rho_{n k m l}-\widetilde{\rho}_{n k m l}\right)(x) G(x-y)\left(\rho_{n k m l}-\widetilde{\rho}_{n k m l}\right)(y) \mathrm{d} x \mathrm{~d} y\right)^{1 / 2},
$$

where $G$ is the periodic Coulomb kernel, solving

$$
-\Delta G(\cdot-y)=4 \pi\left(\delta_{y}-1\right)
$$

with periodic boundary condition on $\Gamma=[0,1]^{d}$ and $\int_{\Gamma} G=0$.

The Coulomb metric is a good measure of the error since density fitting is often used in approximating electron repulsion integral tensor, such as

$$
\begin{aligned}
E_{n k m l} & =\iint_{\Gamma \times \Gamma} \bar{\psi}_{n, k}(x) \psi_{m, l}(x) G(x-y) \bar{\psi}_{m, l}(y) \psi_{n, k}(y) \mathrm{d} x \mathrm{~d} y \\
& =\iint_{\Gamma \times \Gamma} \bar{u}_{n, k}(x) u_{m, l}(x) G(x-y) \bar{u}_{m, l}(y) u_{n, k}(y) e^{-i(k-l) \cdot(x-y)} \mathrm{d} x \mathrm{~d} y .
\end{aligned}
$$

Using the density fitting [4, we get the approximation

$$
\begin{aligned}
\widetilde{E}_{n k m l} & =\sum_{\mu \nu} \iint_{\Gamma \times \Gamma} \bar{C}_{n, k}^{\mu} C_{m, l}^{\mu} P_{\mu}(x) G(x-y) \bar{C}_{m, l}^{v} C_{n, k}^{v} P_{v}(y) e^{-i(k-l) \cdot(x-y)} \mathrm{d} x \mathrm{~d} y \\
& =\sum_{\mu \nu} \bar{C}_{m, k}^{\mu} C_{n, l}^{\mu} \bar{C}_{m, l}^{v} C_{n, k}^{v} \iint_{\Gamma \times \Gamma} P_{\mu}(x) G(x-y) P_{v}(y) e^{-i(k-l) \cdot(x-y)} \mathrm{d} x \mathrm{~d} y .
\end{aligned}
$$


A simple calculation gives

$$
\begin{aligned}
\left|E_{n k m l}-\widetilde{E}_{n k m l}\right|= & \left|\iint\left(\rho_{n k m l}(x) \bar{\rho}_{n k m l}(y)-\widetilde{\rho}_{n k m l}(x) \overline{\tilde{\rho}}_{n k m l}(y)\right) G(x-y) e^{-i(k-l) \cdot(x-y)} \mathrm{d} x \mathrm{~d} y\right| \\
\leq & \left|\iint\left(\rho_{n k m l}(x)-\widetilde{\rho}_{n k m l}(x)\right) \bar{\rho}_{n k m l}(y) G(x-y) e^{-i(k-l) \cdot(x-y)} \mathrm{d} x \mathrm{~d} y\right| \\
& \quad+\left|\iint \widetilde{\rho}_{n k m l}(x)\left(\bar{\rho}_{n k m l}(y)-\overline{\tilde{\rho}}_{n k m l}(y)\right) G(x-y) e^{-i(k-l) \cdot(x-y)} \mathrm{d} x \mathrm{~d} y\right| \\
\leq & C\left\|\rho_{n k m l}-\widetilde{\rho}_{n k m l}\right\|_{C}\left(\left\|\rho_{n k m l}\right\|_{C}+\left\|\widetilde{\rho}_{n k m l}\right\|_{C}\right),
\end{aligned}
$$

where the last step uses the Fourier representation and the Cauchy-Schwartz inequality.

3.1. 2D examples. We consider two examples in two dimensions. In both examples, the periodic model potential $V(x)$ is given by

$$
V(x)=\sum_{n_{1} \in \mathbb{Z}} \sum_{n_{2} \in \mathbb{Z}} V_{c}\left(x-n_{1} e_{1}-n_{2} e_{2}\right)
$$

where $V_{c}$ is a localized potential centered at the origin and $e_{1}$ and $e_{2}$ are the unit Cartesian basis vectors. This is a simple square lattice and the Brillouin zone is also a square. The potential $V_{c}(\cdot)$ is chosen to be centered at the origin and spherically symmetric. The unit cell $[0,1)^{2}$ is discretized with $N_{\text {grid }}=48$ points in each dimension. In each example, we consider up to $N=41$ bands and the Brillouin zone $[-\pi, \pi)^{2}$ is sampled with up to $K=16$ points per dimension. The band structure and the eigenfunctions are computed with spectral discretization for high order accuracy and the resulting discrete eigenvalue problem for each $k$ point is solved using LOBPCG 5 with the simple inverse Laplacian preconditioning. The prescribed accuracy for the column selection algorithm is set to be of order $10^{-5}$.

In the first example, the potential $V_{c}(x)$ is given by a Gaussian profile

$$
V_{c}(x)=-144 \exp \left(-\frac{\|x\|^{2}}{2 \sigma^{2}}\right)
$$

with $\sigma=0.1333$. Figure 1 summarizes the results for this first example.

- Figure 1 (a) shows the potential profile in the unit cell $[-1 / 2,1 / 2)^{2}$.

- Figure 1 (b) gives the band structure along the standard $\Gamma-X-M$ path in the Brillouin zone for simple square lattice.

- Figure 1(c) plots the dependence of $N_{\text {col }}$ (the number of auxiliary basis) as a function of $N$ (the number of bands) for different choices of $K$ (the number of $k$-point samples in each dimension) at the targeted accuracy level $10^{-5}$. From this plot, one can clearly see that $N_{\text {col }}$ grows roughly linearly with respect to $N$ and that it depends only mildly on the number of $k$ point samples.

- The time for performing the column selection in seconds is given in Figure 1(d) as a function of $N$ for different values of $K$. This plot shows that the complexity depends quadratically on $N$ but only grows mildly with $K$. 
- Finally, Figures 1 (e) and (f) give the relative errors in the $L^{2}$ metric and the Coulomb metric, respectively. These two plots show that the estimated relative errors are bounded by a small constant times the prescribed accuracy level.

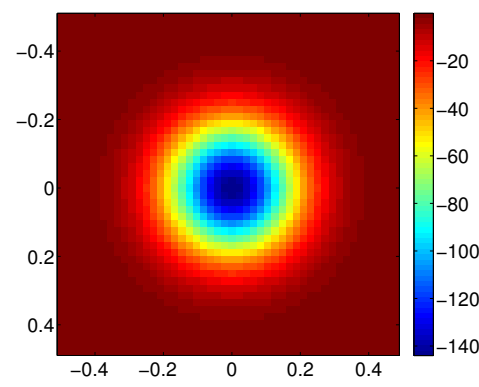

(a)

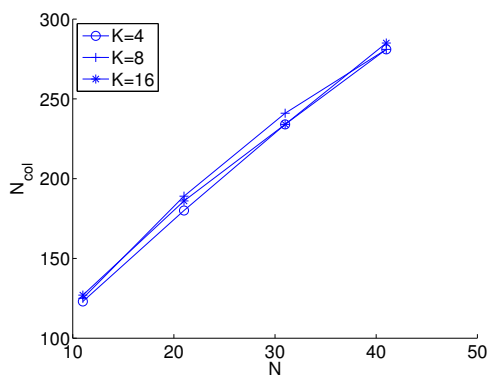

(c)

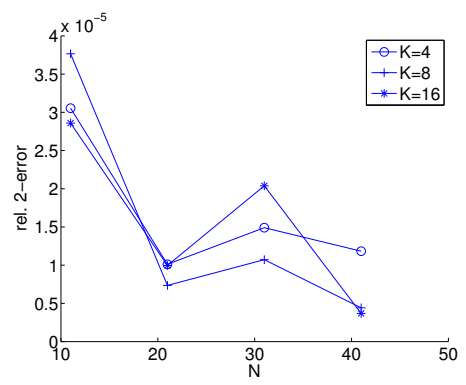

(e)

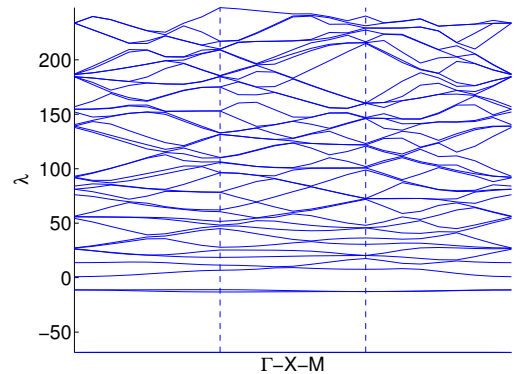

(b)

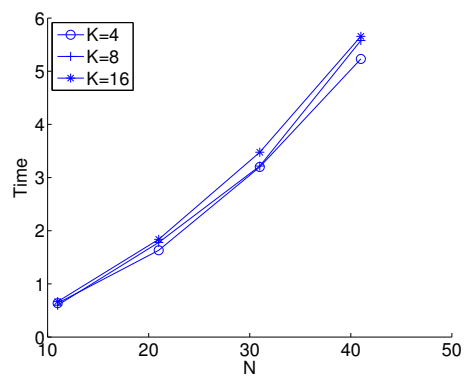

(d)

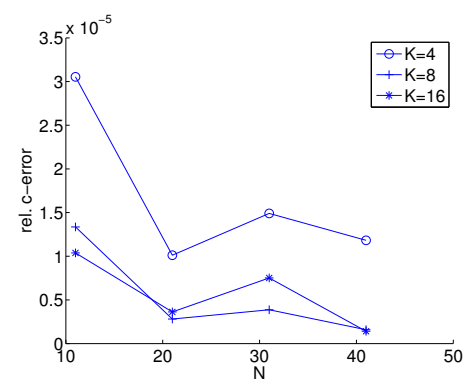

(f)

Figure 1. Results of the first 2D example. (a) the potential $V_{c}$ in unit cell. (b) the band structure plotted along the $\Gamma-X-M$ path. (c) $N_{\text {col }}$ as a function $N$ (the number of bands) for different values of $K$ (the number of $k$ points). (d) The time used by the column selection algorithm in seconds. (e) The relative error measured in the $L^{2}$ metric. (f) The relative error measured in the Coulomb metric.

In the second example, the potential $V_{c}(x)$ is chosen to be

$$
V_{c}(x)=-144 \exp \left(-\frac{\max (\|x\|-1 / 4,0)^{2}}{2 \sigma^{2}}\right)
$$


with $\sigma=0.0667$. The results for this example are summarized in Figure2 The results show similar characteristics as the previous example.

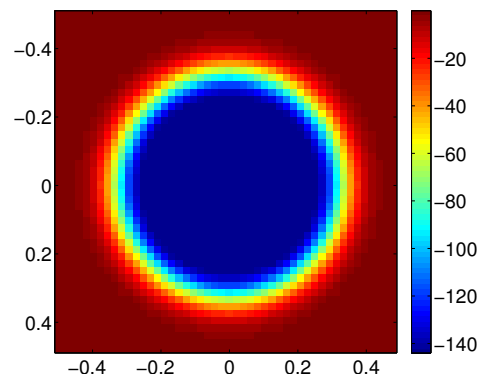

(a)

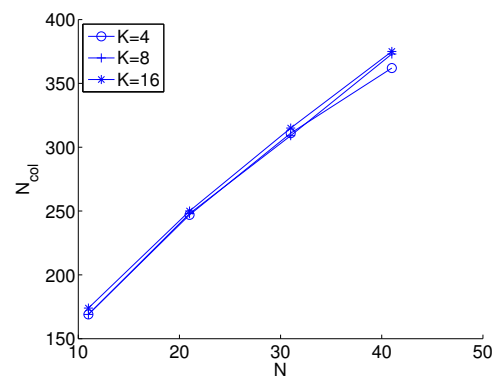

(c)

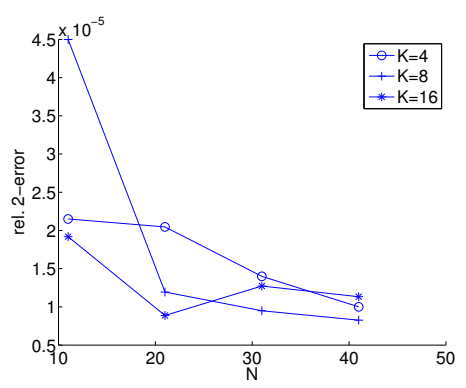

(e)

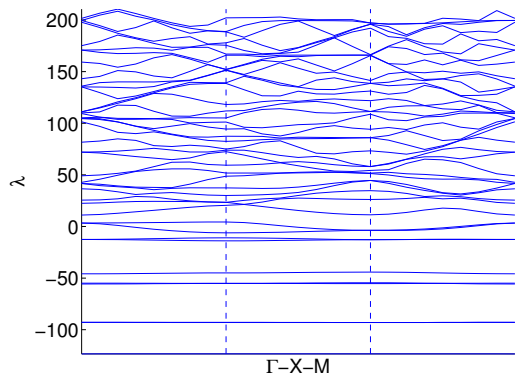

(b)

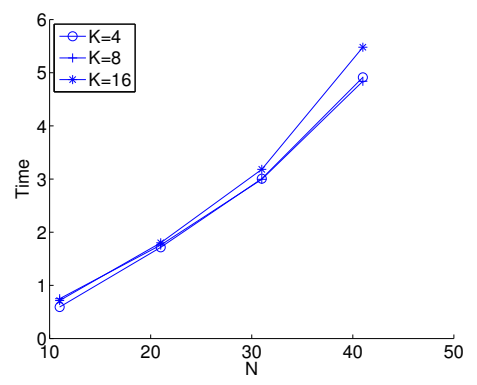

(d)

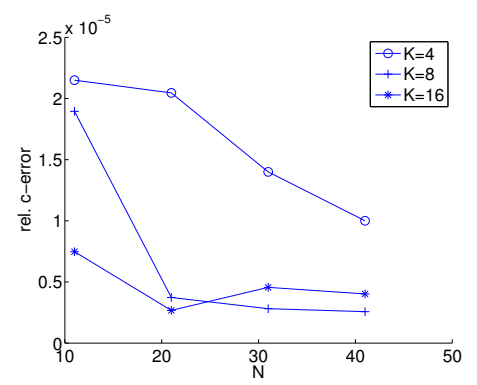

(f)

Figure 2. Results of the second 2D example. (a) the potential $V_{c}$ in unit cell. (b) the band structure plotted along the $\Gamma-X-M$ path. (c) $N_{\text {col }}$ as a function $N$ (the number of bands) for different values of $K$ (the number of $k$ points). (d) The time used by the column selection algorithm in seconds. (e) The relative error measured in the $L^{2}$ metric. (f) The relative error measured in the Coulomb metric.

3.2. 3D examples. For the $3 \mathrm{D}$ case, we consider two similar examples. In each example, the periodic model potential $V(x)$ is given by

$$
V(x)=\sum_{n_{1} \in \mathbb{Z}} \sum_{n_{2} \in \mathbb{Z}} \sum_{n_{3} \in \mathbb{Z}} V_{c}\left(x-n_{1} e_{1}-n_{2} e_{2}-n_{3} e_{3}\right)
$$


where $V_{c}$ is a localized potential centered at the origin and $e_{1}, e_{2}$, and $e_{3}$ are the unit Cartesian basis vectors. This is a simple cubic lattice and the Brillouin zone is also a cube. The potential $V_{c}(\cdot)$ is again chosen to be centered at the origin and spherically symmetric. The unit cell $[0,1)^{3}$ is discretized with $N_{\text {grid }}=24$ points in each dimension. In each example, we consider up to $N=41$ bands and the Brillouin zone $[-\pi, \pi)^{3}$ is sampled with up to $K=12$ points per dimension. Similar to the 2D examples, the band structure and the eigenfunctions are computed with pseudo-spectral discretization for high order accuracy and the resulting discrete eigenvalue problem for each $k$ point is solved using LOBPCG with simple inverse Laplacian preconditioning. The prescribed accuracy for the column selection algorithm is set to be of order $10^{-5}$.

The potential $V_{c}(x)$ in the first example is given by (19) but now in 3D and with $\sigma=0.1667$. Figure 3 summarizes the results for this examples. The meaning of each plot is similar to the ones of the 2D examples, except that

- Figure 3 (a) is the cross-section of the potential $V_{c}(x)$ taken at the plane $x_{3}=0$;

- Figure 3 (b) gives the band structure along the standard $\Gamma-X-M-\Gamma-R-X \mid M-R$ path in the Brillouin zone for the simple cubic lattice.

Similar to the results from the 2D examples, we can draw the following conclusions:

- $N_{\text {col }}$ depends roughly linearly on $N$ but is almost independent of $K$;

- the time of the column selection algorithm grows quadratically in $N$ but is almost independent of $K$;

- the relative errors of the algorithm are bounded by a small factor times the accuracy level used in the column selection algorithm both in the $L^{2}$ metric and the Coulomb metric.

In the second example, the potential $V_{c}(x)$ given by 20) but again interpreted in $3 \mathrm{D}$ and with $\sigma=0.0833$. The results are summarized in Figure 4 .

\section{CONCLUSION}

We present and demonstrate an efficient algorithm for periodic density fitting for Bloch waves. The proposed algorithm is based on randomized pivoted $Q R$ algorithm with a choice of random projection adapted to the tensor product structure of the matrix. The resulting algorithm is validated in several numerical examples in two and three dimensions.

\section{REFERENCES}

[1] B. I. Dunlap, J. W. D. Connolly, and J. R. Sabin, On first-row diatomic molecules and local density models, J. Chem. Phys. 71 (1979), 4993-4999.

[2] G.H. Golub and C.F. Van Loan, Matrix computations, Fourth, Johns Hopkins Studies in the Mathematical Sciences, Johns Hopkins University Press, Baltimore, MD, 2013.

[3] E. G. Hohenstein, S. I. L. Kokkila, R. M. Parrish, and T. J. Martinez, Quartic scaling second-order approximate coupled cluster singles and doubles via tensor hypercontraction: THC-CC2, J. Chem. Phys. 138 (2013), 124111.

[4] E.G. Hohenstein, R.M. Parrish, and T.J. Martinez, Tensor hypercontraction density fitting. I. Quartic scaling secondand third-order Møller-plesset perturbation theory, J. Chem. Phys. 137 (2012), 044103. 


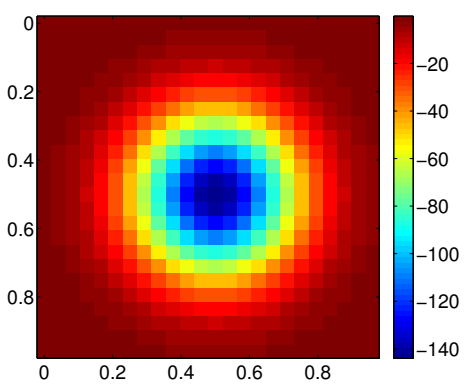

(a)

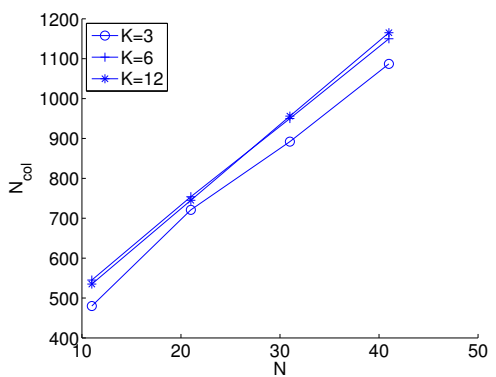

(c)

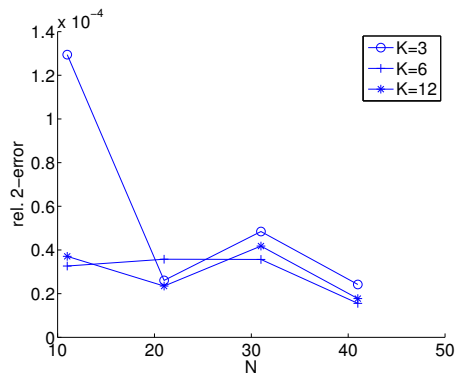

(e)

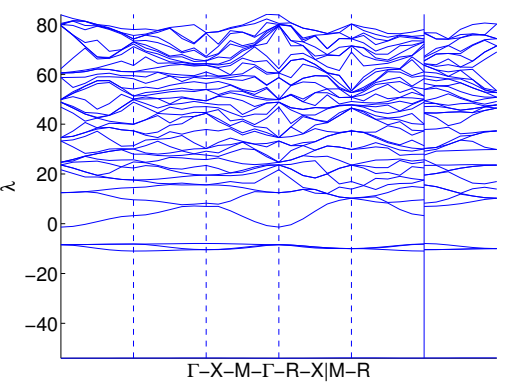

(b)

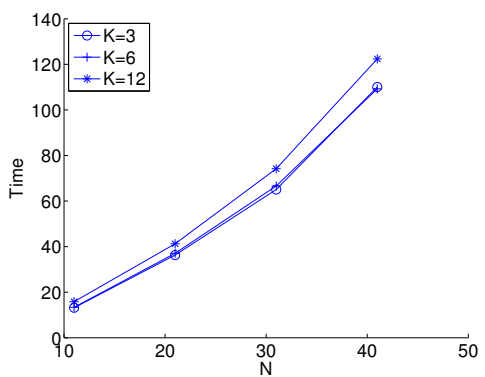

(d)

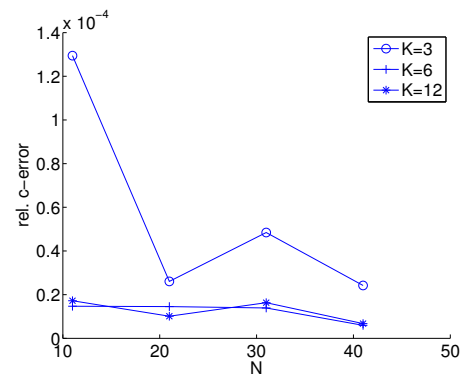

(f)

FIGURE 3. Results of the first 3D example. (a) the cross-section of the potential $V_{c}$ in unit cell taken at $x_{3}=0$. (b) the band structure plotted along the $\Gamma-X-$ $M-\Gamma-R-X \mid M-R$ path. (c) $N_{\text {col }}$ as a function $N$ (the number of bands) for different values of $K$ (the number of $k$ points). (d) The time used by the column selection algorithm in seconds. (e) The relative error measured in the $L^{2}$ metric. (f) The relative error measured in the Coulomb metric.

[5] Andrew V. Knyazev, Toward the optimal preconditioned eigensolver: locally optimal block preconditioned conjugate gradient method, SIAM J. Sci. Comput. 23 (2001), no. 2, 517-541 (electronic). Copper Mountain Conference (2000). MR1861263 (2003g:65050)

[6] S. V. Levchenko, X. Ren, J. Wieferink, R. Johanni, P. Rinke, V. Blum, and M. Scheffler, Hybrid functionals for large periodic systems in an all-electron, numeric atom-centered basis framework, Comp. Phys. Commun. 192 (2015), 6069. 


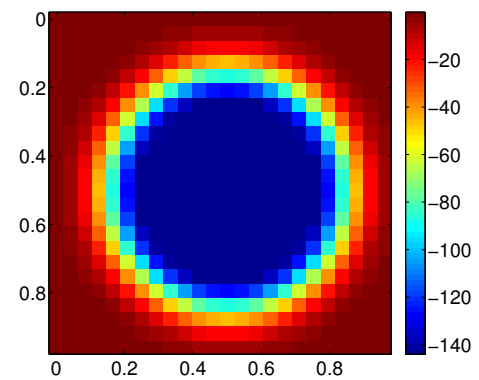

(a)

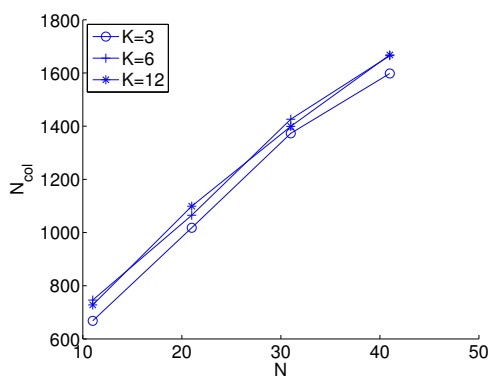

(c)

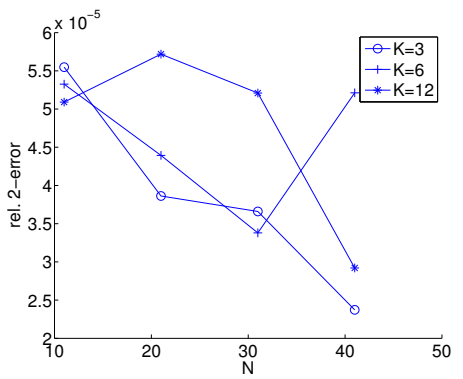

(e)

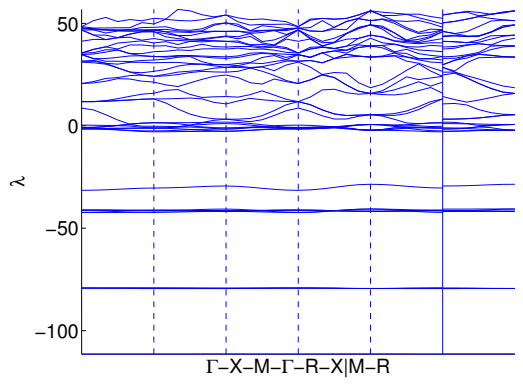

(b)

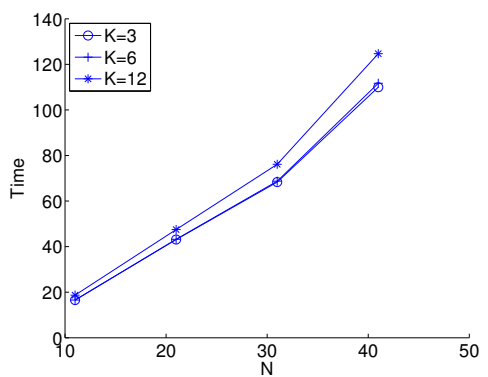

(d)

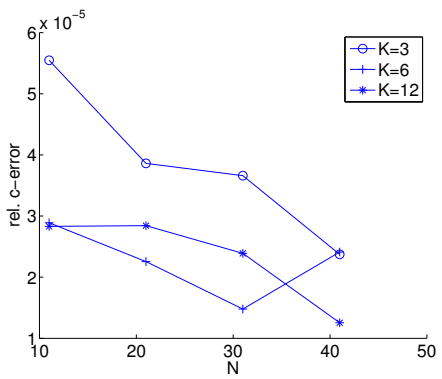

(f)

FIgURE 4. Results of the second 3D example. (a) the cross-section of the potential $V_{c}$ in unit cell taken at $x_{3}=0$. (b) the band structure plotted along the $\Gamma-X-M-\Gamma-R-X \mid M-R$ path. (c) $N_{\text {col }}$ as a function $N$ (the number of bands) for different values of $K$ (the number of $k$ points). (d) The time used by the column selection algorithm in seconds. (e) The relative error measured in the $L^{2}$ metric. (f) The relative error measured in the Coulomb metric.

[7] E. Liberty, F. Woolfe, P.-G. Martinsson, V. Rokhlin, and M. Tygert, Randomized algorithms for the low-rank approximation of matrices, Proc. Natl. Acad. Sci. USA 104 (2007), 20167-20172.

[8] M. Lorenz, D. Usvyat, and M. Schütz, Local ab initio methods for calculating optical band gaps in periodic systems. I. Periodic density fitted local configuration interactino singles method for polymers, J. Chem. Phys. 134 (2011), 094101.

[9] J. Lu and L. Ying, Compression of the electron repulsion integral tensor in tensor hypercontraction format with cubic scaling cost, J. Comput. Phys. 302 (2015), 329-335. 
[10] L. Maschio, D. Usvyat, F. R. Manby, S. Casassa, C. Pisani, and M. Schütz, Fast local-MP2 method with density-fitting for crystals. I. Theory and algorithms, Phys. Rev. B 76 (2007), 075101.

[11] R. M. Parrish, C. David Sherrill, E. G. Hohenstein, S. I. L. Kokkila, and T. J. Martinez, Communication: Acceleration of coupled cluster singles and doubles via orbital-weighted least-squares tensor hypercontraction, J. Chem. Phys. 140 (2014), 181102.

[12] R.M. Parrish, E.G. Hohenstein, T.J. Martinez, and C. David Sherrill, Tensor hypercontraction. II. Least-squares renormalization, J. Chem. Phys. 137 (2012), 224106.

[13] M. Reed and B. Simon, Methods of modern mathematical physics, Vol IV, Academic Press, New York, 1980.

[14] X. Ren, P. Rinke, V. Blum, J. Wieferink, A. Tkatchenko, A. Sanfilippo, K. Reuter, and M. Scheffler, Resolution-of-identity approach to Hartree-Fock, hybrid density functionals, RPA, MP2 and GW with numeric atom-centered orbital basis functions, New J. Phys. 14 (2012), 053020.

[15] M. Schütz, D. Usvyat, M. Lorenz, C. Pisani, L. Maschio, S. Casassa, and M. Halo, Density fitting for correlated calculations in periodic systems, Accurate condensed-phase quantum chemistry, 2010, pp. 27.

[16] N. Shenvi, H. van Aggelen, Y. Yang, and W. Yang, Tensor hypercontracted ppRPA: Reducing the cost of the particleparticle random phase approximation from $O\left(r^{6}\right)$ to $O\left(r^{4}\right)$, J. Chem. Phys. 141 (2014), 024119.

[17] N. Shenvi, H. van Aggelen, Y. Yang, W. Yang, C. Schwerdtfeger, and D. Mazziotti, The tensor hypercontracted parametric reduced density matrix algorithm: Coupled-cluster accuracy with $O\left(r^{4}\right)$ scaling, J. Chem. Phys. 139 (2013), 054110.

[18] A. Sodt, J. E. Subotnik, and M. Head-Gordon, Linear scaling density fitting, J. Chem. Phys. 125 (2006), 194109.

[19] O. Vahtras, J. Almlöf, and M. W. Feyereisen, Integral approximations for LCAO-SCF calculations, Chem. Phys. Lett. 213 (1993), no. 5-6, 514-518.

[20] F. Weigend, M. Häser, H. Patzelt, and R. Ahlrichs, RI-MP2: optimized auxiliary basis sets and demonstration of efficiency, Chem. Phys. Lett. 294 (1998), no. 1-3, 143-152.

[21] F. Woolfe, E. Liberty, V. Rokhlin, and M. Tygert, A fast randomized algorithm for the approximation of matrices, Appl. Comput. Harmon. Anal. 25 (2008), 335 -366.

Department of Mathematics, Department of Physics, and Department of Chemistry, Duke University, Box 90320, DuRHAM NC 27708, USA

E-mail address: jianfeng@math.duke.edu

Department of Mathematics and Institute of Computational and Mathematical Engineering, Stanford University, 450 Serra Mall, Bldg 380, Stanford CA 94305, USA

E-mail address: lexing@stanford.edu 